\title{
Fractional Ulam-stability of fractional impulsive differential equation involving Hilfer-Katugampola fractional differential operator
}

\author{
S. Harikrishnan ${ }^{\mathrm{a}^{*}}$, Rabha W. Ibrahim ${ }^{\mathrm{b}}$ and K. Kanagarajan ${ }^{\mathrm{a}}$ \\ ${ }^{a}$ Department of Mathematics, Sri Ramakrishna Mission Vidyalaya College of Arts and Science, Coimbatore-641020, India \\ ${ }^{\mathrm{b}}$ Faculty of Computer Science and Information Technology, University Malaya, 50603, Malaysia \\ *Corresponding author E-mail: rabhaibrahim@yahoo.com
}

\section{Article Info}

Keywords: Fractional calculus, Fractional differential equations, Fractional differential operator.

2010 AMS: $44 A 45$

Received: 28 April 2018

Accepted: 10 June 2018

Available online: 26 June 2018

\begin{abstract}
In this note, we set up existence, uniqueness as well as the stability of a special class of fractional differential equation (FDE) with Hilfer-Katugampola fractional differential operator (HKFDO). The outcomes are given by employing the Schaefer's fixed point theorem and Banach contraction principle. Moreover, we modify the fractional Ulam stability (FUS) concept utilizing HKFDO.
\end{abstract}

\section{Introduction}

The idea of impulsive differential equations has had attention many investigators. Its developments over more than twenty years in almost all science. It performed as an essential role in present day in current applied mathematical model of real techniques bobbing up in phenomena studied in physics, chemical generation, population studies and political economy; one can follow the monograph of Lakshmikantham et al. [12]. The analysis of impulsive differential equation involving classical derivatives one can refer to [2, 13, 14, 16]. Nowadays the investigation of FDE involving Hilfer fractional operator introduced by Hilfer [4] is increasing rapidly one can refer to [3, 9, 10]. Later on the generalized fractional derivative introduced by U.N. Katugampola [11] is unified with Hilfer fractional derivative by Oliveira and E. Capelas de Oliveira in [15] is named as Hilfer-Katugampola fractional derivative.

The fractional Ulam-Hyers stability (FUHRS) of FDE has been studied in $[5,17]$ utilizing the classical fractional calculus. While, this form of stability has been formalized in a complex domain for the Cauchy problem in [6]-[8]. Here, we shall introduce a generalization for FUHRS involving a multi- power of fractional calculus.

Consider the impulsive differential equation involving Hilfer-Katugampola fractional derivative of the form

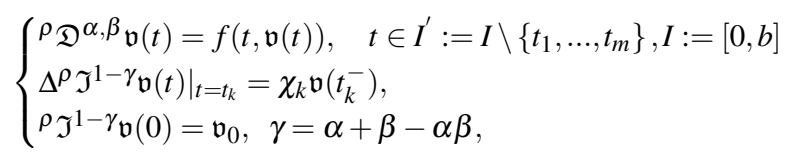

where $\rho^{\rho} \mathfrak{D}^{\alpha, \beta}$ is Hilfer-Katugampola fractional differential operator of order $\alpha(0<\alpha<1), \beta(0 \leq \beta \leq 1), \rho^{\rho} \mathfrak{I}^{1-\gamma}$ is a generalization fractional integral operator of order $1-\gamma, \rho>0, f: I \times R \rightarrow R$ is a given continuous function, $\chi_{k}: R \rightarrow R$, and $0=t_{0}<t_{1}<\ldots<t_{m}<t_{m+1}=b$,

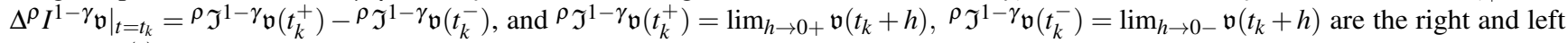
limits of $\mathfrak{v}(t)$ at $t=t_{k}$ respectively.

The paper constructed as follows: In Section 2, we present the main definitions and preliminaries. In Section 3, we deal with the finding results. In Section 4, we introduce a generalization of a special class of FUHRS. 


\section{Preliminaries}

Here, we recall some of well known concepts ( see [1, 13, 15]). Consider the space

$$
P C(I, R)=\left\{\mathfrak{v}: I \rightarrow R: \mathfrak{v}(t) \in C\left(t_{k}, t_{k+1}\right], k=0, \ldots, m ; \text { there exists } \mathfrak{v}\left(t_{k}^{+}\right) \operatorname{and} \mathfrak{v}\left(t_{k}^{-}\right)\right\} .
$$

Now we consider the weighted space $P C_{\gamma}(I, R)$.

$$
P C_{\gamma, \rho}(I, R)=\left\{\mathfrak{v}:\left.\left(\frac{t^{\rho}-t_{k}^{\rho}}{\rho}\right)^{\gamma} \mathfrak{v}\right|_{\left[t_{k}, t_{k+1}\right]} \in C\left[t_{k}, t_{k+1}\right], k=0, \ldots, m \text { where } 0 \leq \gamma<1\right\} .
$$

Obviously, it is a Banach space with norm

$$
\|\mathfrak{v}\|_{P C_{\gamma, \rho}}=\sup _{\left(t_{k}, t_{k+1}\right]}\left\{\left(\frac{t^{\rho}-t_{k}^{\rho}}{\rho}\right)^{\gamma} \mathfrak{v}(t)\right\}, k=0, \ldots, m .
$$

The following spaces are used to solve the problem:

$$
P C_{1-\gamma, \rho}^{\alpha, \beta}(I, R)=\left\{f \in P C_{1-\gamma, \rho}(I, R),{ }^{\rho} \mathfrak{D}^{\alpha, \beta} f \in P C_{\mu, \rho}(I, R)\right\}
$$

and

$$
P C_{1-\gamma, \rho}^{\gamma}(I, R)=\left\{f \in P C_{1-\gamma, \rho}(I, R),{ }^{\rho} \mathfrak{D}^{\gamma} f \in P C_{1-\gamma, \rho}(I, R)\right\} .
$$

It is obvious that

$$
P C_{1-\gamma, \rho}^{\gamma}(I, R) \subset P C_{1-\gamma, \rho}^{\alpha, \beta}(I, R) .
$$

Definition 2.1. The generalized left-sided fractional integral ${ }^{\rho} I_{a^{+}}^{\alpha}$ f of order $\alpha \in C(\Re(\alpha))$ is defined by

$$
\left(\rho_{\mathfrak{I}_{a^{+}}^{\alpha}}^{\alpha}\right) f(t)=\frac{\rho^{1-\alpha}}{\Gamma(\alpha)} \int_{a}^{t}\left(t^{\rho}-s^{\rho}\right)^{\alpha-1} s^{\rho-1} f(s) d s, t>a .
$$

The generalized fractional differential operator, corresponding to the generalized fractional integral operator (2.1), is defined for $0 \leq a<t$, by

$$
\left({ }^{\rho} \mathfrak{D}_{a^{+}}^{\alpha} f\right)(t)=\frac{\rho^{\alpha-n-1}}{\Gamma(n-\alpha)}\left(t^{1-\rho} \frac{d}{d t}\right)^{n} \int_{a}^{t}\left(t^{\rho}-s^{\rho}\right)^{n-\alpha+1} s^{\rho-1} f(s) d s
$$

if the integral exists.

Definition 2.2. The Hilfer-Katugampola fractional derivative with respect to $t$, with the fractional power $\rho>0$, is defined by

$$
\begin{aligned}
\left(\rho_{\left.\mathfrak{D}_{a^{ \pm}}^{\alpha, \beta} f\right)(t)}\right. & =\left( \pm^{\rho} \mathfrak{I}_{a^{ \pm}}^{\alpha}\left(t^{\rho-1} \frac{d}{d t}\right) \rho^{\rho} \mathfrak{I}_{a^{ \pm}}^{(1-\beta)(1-\alpha)}\right)(t) \\
& =\left({ }_{ \pm}^{\rho} \mathfrak{I}_{a^{ \pm}}^{\alpha} \delta_{\rho}{ }^{\rho} \mathfrak{I}_{a^{ \pm}}^{(1-\beta)(1-\alpha)}\right)(t) .
\end{aligned}
$$

- The operator $\rho_{\mathfrak{D}_{a^{+}}^{\alpha, \beta}}^{\alpha a n \text { be written as }}$

$$
\rho_{\mathfrak{D}_{a^{+}}^{\alpha, \beta}}={ }^{\rho} \mathfrak{I}_{a^{+}}^{\beta(1-\alpha)} \delta_{\rho}{ }^{\rho} \mathfrak{I}_{a^{+}}^{1-\gamma}={ }^{\rho} \mathfrak{I}_{a^{+}}^{\beta(1-\alpha)} \rho_{\mathfrak{D}_{a^{+}}^{\gamma}}^{\gamma}, \quad \gamma=\alpha+\beta-\alpha \beta
$$

- The fractional operator $\rho_{\mathfrak{D}_{a^{+}}^{\alpha, \beta}}$ is considered as interpolation, with the convenient parameters, of the following fractional derivatives, Hilfer fractional differential operator when $(\rho \rightarrow 1)$, Hilfer-Hadamard fractional derivative when $(\rho \rightarrow 0)$, generalized fractional derivative when $(\beta=0)$, Caputo-type fractional derivative when $(\beta=1)$, Riemann-Liouville fractional differential operator when $(\beta=0, \rho \rightarrow 1)$, Hadamard fractional operator when $(\beta=0, \rho \rightarrow 0)$, Caputo fractional operator when $(\beta=1, \rho \rightarrow 1)$. CaputoHadamard fractional operator when $(\beta=1, \rho \rightarrow 0)$, Liouville fractional operator when $(\beta=0, \rho \rightarrow 1, a=0)$, Hadamard fractional operator when $(\beta=0, \rho \rightarrow 1, a=-\infty)$, We consider the following parameters $\alpha, \beta, \gamma$ satisfying

$$
\gamma=\alpha+\beta-\alpha \beta, 0 \leq \gamma<1, \alpha>0, \beta<1 .
$$

For $\alpha>0, \beta>0$ and $0 \leq \gamma<1$. The properties are given as follows,

1. If $f \in C_{\gamma}(I, R)$, then we have the following semigroup property

$$
\left({ }^{\rho} \mathfrak{I}^{\alpha \rho} \mathfrak{I}^{\beta} f\right)(t)=\left({ }^{\rho} \mathfrak{I}^{\alpha+\beta}\right)(t) .
$$

2. If $f \in C_{\gamma}(I, R)$, then

$$
\left(\rho \mathfrak{D}^{\alpha \rho} \mathfrak{I}^{\alpha} f\right)(t)=f(t) .
$$


3. If $t>0$ then

$$
\rho_{\mathfrak{I}^{\alpha}}\left(\frac{t^{\rho}}{\rho}\right)^{\beta-1}(t)=\frac{\Gamma(\beta)}{\Gamma(\alpha+\beta)}\left(\frac{t^{\rho}}{\rho}\right)^{\alpha+\beta-1}
$$

and

$$
\rho_{\mathfrak{D}^{\alpha}}\left(\frac{t^{\rho}}{\rho}\right)^{\beta-1}(t)=0
$$

4. If $f \in P C_{\gamma}$ and ${ }^{\rho} \mathfrak{I}^{1-\alpha} f \in P C_{\gamma}^{1}(I, R)$, then

$$
\left({ }^{\rho} \mathfrak{I}^{\alpha \rho} \mathfrak{D}^{\alpha}\right)(t)=f(t)-\frac{\left({ }^{\rho} \mathfrak{I}^{1-\alpha} f\right)(0)}{\Gamma(\alpha)}\left(\frac{t^{\rho}}{\rho}\right)^{\alpha-1},
$$

5. If $\alpha>\gamma$, then ${ }^{\rho} \mathfrak{I}^{\alpha} f$ is continuous on $[0, b]$

$$
\rho_{\mathfrak{I}^{\alpha}} f(0)=\lim _{t \rightarrow 0}{ }^{\rho} \mathfrak{I}^{\alpha} f(t)=0 .
$$

6. If $f \in P C_{\gamma, \rho}^{\gamma}(I, R)$, then

$$
\rho_{\mathfrak{I}^{\gamma}} \mathfrak{D}^{\gamma} f(t)={ }^{\rho} \mathfrak{I}^{\alpha} \rho_{\mathfrak{D}^{\alpha, \beta}} f(t)
$$

and

$$
\rho_{\mathfrak{D}^{\gamma \rho}} \mathfrak{I}^{\alpha} f(t)=\rho_{\mathfrak{D}^{\beta(1-\alpha)}} f(t) .
$$

7. Let $f \in L^{1}(0, b)$. If ${ }^{\rho} \mathfrak{D}^{\beta(1-\alpha)} f$ occurs on $L^{1}(0, b)$, then

$$
\rho_{\mathfrak{D}^{\alpha, \beta}} \rho_{\mathfrak{I}^{\alpha}}^{\alpha} f(t)={ }^{\rho} \mathfrak{I}^{\beta(1-\alpha)} \rho_{\mathfrak{D}^{\beta(1-\alpha)}} f(t) .
$$

8. If $f \in P C_{\gamma, \rho}(I, R)$ and ${ }^{\rho} \mathfrak{I}^{1-\beta(1-\alpha)} \in P C_{1-\gamma}^{1}(I, R)$, then ${ }^{\rho} \mathfrak{D}^{\alpha, \beta} \mathfrak{I}^{\alpha}$ exists on $[0, b]$ and

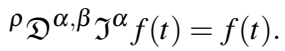

Lemma 2.3. Let $\mathfrak{v} \in P C_{1-\gamma}(I, R)$ satisfies the following inequality

$$
|\mathfrak{v}(t)| \leq c_{1}+c_{2} \int_{0}^{t}\left(\frac{t^{\rho}-s^{\rho}}{\rho}\right)^{\alpha-1} s^{\rho-1}|\mathfrak{v}(t)| d s+\sum_{0<t_{k}<t} \chi_{k}\left|\mathfrak{v}\left(t_{k}\right)\right|
$$

where $c_{1}$ is a non-negative, continuous and non-decreasing function on I and $c_{2}, \chi_{i}$ are constants. Then

$$
|\mathfrak{v}(t)| \leq c_{1}\left(1+\chi E_{\alpha}\left(c_{2} \Gamma(\alpha) t^{\alpha}\right)^{k} E_{\alpha}\left(c_{2} \Gamma(\alpha) t^{\alpha}\right) \text { for } t \in\left(t_{k} \cdot t_{k+1}\right],\right.
$$

where $\chi=\sup \left\{\chi_{k}: k=1,2,3, \ldots,\right\}$.

Theorem 2.4. (Schaefer's fixed point theorem) Let $\mathfrak{P}: K \rightarrow K$ be completely continuous operator. If set $E[\mathfrak{P}]=\{\mathfrak{v} \in K: \mathfrak{v}=\delta(\mathfrak{P v})$, for some $\delta \in[0, b]$ is bounded, Then $\mathfrak{P}$ has fixed point.

Lemma 2.5. A function $\mathfrak{v}$ is the solution of fractional impulsive differential equation

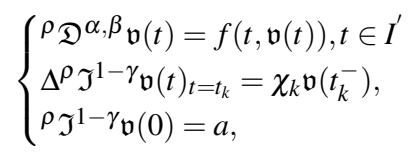

if and only if $\mathfrak{v}$ achieves the integral equation

$$
\mathfrak{v}(t)=\left(\frac{t^{\rho}-t_{k}^{\rho}}{\rho}\right)^{\gamma-1} \frac{1}{\Gamma(\gamma)}\left[a+\sum_{0<t_{k}<t} \chi_{k} \mathfrak{v}\left(t_{k}\right)+\sum_{0<t_{k}<t} \rho_{\mathfrak{I}_{t_{k-1}}^{1-\beta(1-\alpha)}} f\left(t_{k}, \mathfrak{v}\left(t_{k}\right)\right)\right]+{ }^{\rho} \mathfrak{I}_{t_{k}}^{\alpha} f(t, \mathfrak{v}(t)) .
$$




\section{Findings}

We make the following hypotheses to prove our main results.

(H1) Let $f: I \times R \longrightarrow R$ be a continuous function and a positive constant $L>0$ accomplishing $|f(t, \mathfrak{v})-f(t, \overline{\mathfrak{v}})| \leq L|\mathfrak{v}-\overline{\mathfrak{v}}|$, for all $\mathfrak{v}, \overline{\mathfrak{v}} \in R$.

(H2) Let $f: I \times R \rightarrow R$ be a completely continuous function and a function $\mu \in L^{1}$ fulfilling $|f(t, \mathfrak{v})| \leq|\mu(t)|$, for all $t \in I, \mathfrak{v} \in R$.

(H3) Let the functions $\chi_{k}: R \rightarrow R$ be continuous and a constant $L_{k}^{*}>0$ achieving

$$
\left|\chi_{k}\left(\mathfrak{v}\left(t_{k}^{-}\right)\right)-\chi_{k}\left(\overline{\mathfrak{v}}\left(t_{k}^{-}\right)\right)\right| \leq L_{k}^{*}\left|\mathfrak{v}\left(t_{k}\right)-\overline{\mathfrak{v}}\left(t_{k}\right)\right|, \text { for all } \mathfrak{v}, \overline{\mathfrak{v}} \in R, k=1,2, \ldots, m
$$

(H4) Let the functions $\chi_{k}: R \rightarrow R$ be continuous and a constant $\mu \in L^{1}$ satisfying

$$
\left|\chi_{k}\left(\mathfrak{v}\left(t_{k}^{-}\right)\right)\right| \leq\left|\mu^{*}(t)\right|, \text { for all } \mathfrak{v} \in R, k=1,2, \ldots, m \text {. }
$$

(H5) There is an increasing function $\varphi \in P C_{1-\gamma, \rho}(I, R)$ and there occurs $\lambda_{\varphi}>0$ such that for any $t \in I$

$$
\rho_{\mathfrak{I}^{\alpha}}^{\alpha} \varphi(t) \leq \lambda_{\varphi} \varphi(t)
$$

Theorem 3.1. Assume that [H1] - [H4] are satisfied. Then, Eq.(1.1) has at least one solution.

Proof. The proof will be given in several steps.

Consider the operator $\mathfrak{P}: P C_{1-\gamma, \rho}(I, R) \rightarrow P C_{1-\gamma, \rho}(I, R)$. The equivalent integral Eq. (2.6) which can be written in the operator form

$$
\mathfrak{v}(t)=\mathfrak{P} \mathfrak{v}(t)
$$

where

$$
\begin{gathered}
\mathfrak{P v}(t)=\left(\frac{t^{\rho}-t_{k}^{\rho}}{\rho}\right)^{\gamma-1} \frac{1}{\Gamma(\gamma)}\left[a+\sum_{0<t_{k}<t} \chi_{k} \mathfrak{v}\left(t_{k}\right)+\sum_{0<t_{k}<t} \rho_{\left.\mathfrak{I}_{t_{k-1}}^{1-\beta(1-\alpha)} f\left(t_{k}, \mathfrak{v}\left(t_{k}\right)\right)\right]}+{ }_{\mathfrak{I}_{t_{k}}^{\alpha} f(t, \mathfrak{v}(t)) .}\right.
\end{gathered}
$$

We shall show that the operator $\mathfrak{P}$ is continuous and completely continuous.

Claim 1: $\mathfrak{P}$ is continuous.

Let $\mathfrak{v}_{n}$ be a sequence such that $\mathfrak{v}_{n} \rightarrow \mathfrak{v}$ in $P C_{1-\gamma, \rho}(I, R)$. Then for each $t \in I$,

$$
\begin{aligned}
\left|\left(\left(\mathfrak{P} \mathfrak{v}_{n}\right)(t)-(\mathfrak{P v})(t)\right)\left(\frac{t^{\rho}-t_{k}^{\rho}}{\rho}\right)^{1-\gamma}\right| & \leq \frac{1}{\Gamma(\gamma)}\left[\sum_{0<t_{k}<t}\left|\chi_{k}\left(\mathfrak{v}_{n}\left(t_{k}\right)\right)-\chi_{k}\left(\mathfrak{v}\left(t_{k}\right)\right)\right|+\sum_{0<t_{k}<t} \mathfrak{I}_{t_{k-1}}^{1-\beta(1-\alpha)}\left|f\left(t_{k}, \mathfrak{v}_{n}\left(t_{k}\right)\right)-f\left(t_{k}, \mathfrak{v}\left(t_{k}\right)\right)\right|\right] \\
& +\left(\frac{t^{\rho}-t_{k}^{\rho}}{\rho}\right)^{1-\gamma} \mathfrak{I}_{t_{k}}^{\alpha}\left|f\left(t, \mathfrak{v}_{n}(t)\right)-f(t, \mathfrak{v}(t))\right|,
\end{aligned}
$$

since $\mathrm{f}$ is continuous, then we have

$$
\left\|\left(\mathfrak{P} \mathfrak{v}_{n}\right)(t)-(\mathfrak{P v})(t)\right\|_{P C_{1-\gamma, \rho}} \rightarrow 0 \text { as } n \rightarrow \infty .
$$

Claim 2: We show that $\mathfrak{P}$ is the mapping of two bounded set.

For $r>0$, there exists a positive constant $l$ such that

$B_{r}=\left\{\mathfrak{v} \in P C_{1-\gamma, \rho}(I, R):\|\mathfrak{v}\|_{P C_{1-\gamma}} \leq r\right\}$, we have $\|(N \mathfrak{v})\|_{P C_{1-\gamma, \rho}} \leq l$.

$$
\begin{aligned}
\left|(\mathfrak{P v})(t)\left(\frac{t^{\rho}-t_{k}^{\rho}}{\rho}\right)^{1-\gamma}\right| \leq & \frac{1}{\Gamma(\gamma)}\left[a+\sum_{0<t_{k}<t}\left|\chi_{k}\left(\mathfrak{v}\left(t_{k}\right)\right)\right|+\sum_{0<t_{k}<t} \mathfrak{I}_{t_{k-1}}^{1-\beta(1-\alpha)}\left|f\left(t_{k}, \mathfrak{v}\left(t_{k}\right)\right)\right|+\left(\frac{t^{\rho}-t_{k}^{\rho}}{\rho}\right)^{1-\gamma} \mathfrak{I}_{t_{k}}^{\alpha}|f(t, \mathfrak{v}(t))|\right. \\
\leq & \frac{1}{\Gamma(\gamma)}\left[a+m\left(\frac{t^{\rho}-t_{k}^{\rho}}{\rho}\right)^{\gamma-1}\left\|\mu^{*}(t)\right\|_{P C_{1-\gamma, \rho}}+\|\mu(t)\|_{P C_{1-\gamma, \rho}}\left(\frac{t_{k}^{\rho}-t_{k-1}^{\rho}}{\rho}\right)^{\alpha} \frac{m B(\gamma, 1-\beta(1-\alpha))}{\Gamma(1-\beta(1-\alpha))}\right] \\
& +\left(\frac{t^{\rho}-t_{k}^{\rho}}{\rho}\right)^{1-\gamma} \frac{B(\gamma, \alpha)}{\Gamma(\alpha)}\left(\frac{t^{\rho}-t_{k}^{\rho}}{\rho}\right)^{\alpha+\gamma-1}\|\mu(t)\|_{P C_{1-\gamma, \rho}} \\
\leq & \frac{1}{\Gamma(\gamma)}\left[a+m\left(\frac{b^{\rho}}{\rho}\right)^{\gamma-1}\left\|\mu^{*}(t)\right\|_{P C_{1-\gamma, \rho}}+\frac{m B(\gamma, 1-\beta(1-\alpha))}{\Gamma(1-\beta(1-\alpha))}\left(\frac{b^{\rho}}{\rho}\right)^{\alpha}\|\mu(t)\|_{P C_{1-\gamma, \rho}}\right] \\
& +\frac{B(\gamma, \alpha)}{\Gamma(\alpha)}\left(\frac{b^{\rho}}{\rho}\right)^{\alpha}\|\mu(t)\|_{P C_{1-\gamma, \rho}} \\
= & l .
\end{aligned}
$$


Claim 3: We show that $\mathfrak{P}$ maps bounded sets into equicontinuous set.

Let $t_{1}, t_{2} \in I, t_{1}<t_{2}, B_{r}$ be a bounded set of $P C_{1-\gamma, \rho}(I, R)$ as in Claim 2, and $\mathfrak{v} \in B_{r}$. Then,

$$
\begin{aligned}
& \left|(\mathfrak{P} \mathfrak{v})\left(t_{1}\right)\left(\frac{t_{1}^{\rho}-t_{k}^{\rho}}{\rho}\right)^{1-\gamma}-(\mathfrak{P v})\left(t_{2}\right)\left(\frac{t_{2}^{\rho}-t_{k}^{\rho}}{\rho}\right)^{1-\gamma}\right| \\
& \leq \frac{1}{\Gamma(\gamma)}\left[a+\sum_{0<t_{k}<t_{1}} \chi_{k} \mathfrak{v}\left(t_{k}\right)+\sum_{0<t_{k}<t_{1}} \rho_{\mathfrak{I}_{t_{k-1}}^{1-\beta(1-\alpha)}} f\left(t_{k}, \mathfrak{v}\left(t_{k}\right)\right)\right]+\left(\frac{t_{1}^{\rho}-t_{k}^{\rho}}{\rho}\right)^{1-\gamma} \rho_{\mathfrak{I}_{t_{k}}^{\alpha}}^{\alpha} f\left(t_{1}, \mathfrak{v}\left(t_{1}\right)\right) \\
& -\frac{1}{\Gamma(\gamma)}\left[a+\sum_{0<t_{k}<t_{2}} \chi_{k} \mathfrak{v}\left(t_{k}\right)+\sum_{0<t_{k}<t_{2}} \rho_{\mathfrak{I}_{t_{k-1}}^{1-\beta(1-\alpha)}} f\left(t_{k}, \mathfrak{v}\left(t_{k}\right)\right)\right]-\left(\frac{t_{2}^{\rho}-t_{k}^{\rho}}{\rho}\right)^{1-\gamma} \rho_{\mathfrak{I}_{t_{k}}^{\alpha} f\left(t_{2}, \mathfrak{v}\left(t_{2}\right)\right)} \\
& \leq \frac{1}{\Gamma(\gamma)}\left[\sum_{0<t_{k}<t_{1}-t_{2}} \chi_{k} \mathfrak{v}\left(t_{k}\right)+\sum_{0<t_{k}<t_{1}-t_{2}} \rho_{t_{k-1}}^{1-\beta(1-\alpha)} f\left(t_{k}, \mathfrak{v}\left(t_{k}\right)\right)\right] \\
& +\|f\|_{P C_{1-\gamma, \rho}} \frac{B(\gamma, \alpha)}{\Gamma(\alpha)}\left|\left(\frac{t_{1}^{\rho}-t_{k}^{\rho}}{\rho}\right)^{\alpha}-\left(\frac{t_{2}^{\rho}-t_{k}^{\rho}}{\rho}\right)^{\alpha}\right|
\end{aligned}
$$

As $t_{1} \rightarrow t_{2}$, the right hand side of the above inequality tends to zero. From Claim 1 to 3 , together with Arzela-Ascoli theorem, we conclude that $\mathfrak{P}: P C_{1-\gamma, \rho}(I, R) \rightarrow P C_{1-\gamma, \rho}(I, R)$ is continuous and completely continuous.

Claim 4: A priori bounds.

Now we prove that

$$
\omega=\left\{\mathfrak{v} \in P C_{1-\gamma, \rho}(I, R): \mathfrak{v}=\delta N(\mathfrak{v}), 0<\delta<1\right\}
$$

is bounded set.

Let $\mathfrak{v} \in \omega, \mathfrak{v}=\delta \mathfrak{P}(\mathfrak{v})$ for some $0<\delta<1$. Thus for each $t \in I$. We have

$$
\mathfrak{v}(t)=\delta\left[\left(\frac{t^{\rho}-t_{k}^{\rho}}{\rho}\right)^{\gamma-1} \frac{1}{\Gamma(\gamma)}\left[a+\sum_{0<t_{k}<t} \chi_{k} \mathfrak{v}\left(t_{k}\right)+\sum_{0<t_{k}<t} \rho^{\rho} \mathfrak{I}_{t_{k-1}}^{1-\beta(1-\alpha)} f\left(t_{k}, \mathfrak{v}\left(t_{k}\right)\right)\right]+{ }^{\rho} \mathfrak{I}_{t_{k}}^{\alpha} f(t, \mathfrak{v}(t))\right] .
$$

We show this Claim by letting the estimation in Claim 2. Finally, by Theorem 2.4, we deduce that $\mathfrak{P}$ has a fixed point and it is the solution of problem (1.1).

Theorem 3.2. Assume that the hypothesis (H1) and (H3) are fulfilled. If

$$
\left[\frac{1}{\Gamma(\gamma)}\left(m L^{*}\left(\frac{b^{\rho}}{\rho}\right)^{\gamma-1}+\frac{m L B(\gamma, 1-\beta(1-\alpha))}{\Gamma(1-\beta(1-\alpha))}\left(\frac{b^{\rho}}{\rho}\right)^{\alpha}\right)+\frac{L B(\gamma, \alpha)}{\Gamma(\alpha)}\left(\frac{b^{\rho}}{\rho}\right)^{\alpha}\right]<1
$$

then, Eq. (1.1) has a unique solution.

Proof. Let $\mathfrak{v}, \overline{\mathfrak{v}} \in P C_{1-\gamma, \rho}(I, R)$ and $t \in I$, then we have

$$
\begin{aligned}
& \left|(\mathfrak{P} \mathfrak{v}(t)-\mathfrak{P} \overline{\mathfrak{v}}(t))\left(\frac{t^{\rho}-t_{k}^{\rho}}{\rho}\right)^{1-\gamma}\right|
\end{aligned}
$$

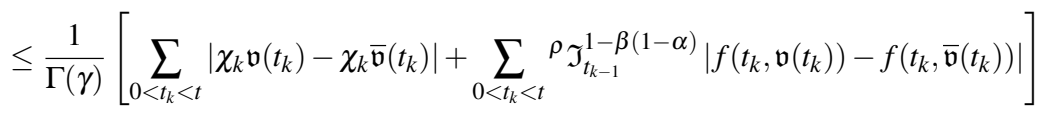

$$
\begin{aligned}
& +\left(\frac{t^{\rho}-t_{k}^{\rho}}{\rho}\right)^{1-\gamma} \rho_{\mathfrak{I}_{t_{k}}^{\alpha}}|f(t, \mathfrak{v}(t))-f(t, \overline{\mathfrak{v}}(t))| \\
& \leq \frac{1}{\Gamma(\gamma)}\left[m L^{*}\left(\frac{t^{\rho}-t_{k}^{\rho}}{\rho}\right)^{\gamma-1}\|\mathfrak{v}-\overline{\mathfrak{v}}\|_{P C_{1-\gamma, \rho}}+\frac{m L B(\gamma, 1-\beta(1-\alpha))}{\Gamma(1-\beta(1-\alpha))}\left(\frac{t_{k}^{\rho}-t_{k-1}^{\rho}}{\rho}\right)^{\alpha}\|\mathfrak{v}-\overline{\mathfrak{v}}\|_{P C_{1-\gamma, \rho}}\right] \\
& +\left(\frac{t^{\rho}-t_{k}^{\rho}}{\rho}\right)^{1-\gamma} \frac{L B(\gamma, \alpha)}{\Gamma(\alpha)}\left(\frac{t^{\rho}-t_{k}^{\rho}}{\rho}\right)^{\alpha+\gamma-1}\|\mathfrak{v}-y\|_{P C_{1-\gamma, \rho}} \\
& \leq \frac{1}{\Gamma(\gamma)}\left[m L^{*}\left(\frac{b^{\rho}}{\rho}\right)^{\gamma-1}\|\mathfrak{v}-\overline{\mathfrak{v}}\|_{P C_{1-\gamma, \rho}}+\frac{m L B(\gamma, 1-\beta(1-\alpha))}{\Gamma(1-\beta(1-\alpha))}\left(\frac{b^{\rho}}{\rho}\right)^{\alpha}\|\mathfrak{v}-\overline{\mathfrak{v}}\|_{P C_{1-\gamma, \rho}}\right] \\
& +\frac{L B(\gamma, \alpha)}{\Gamma(\alpha)}\left(\frac{b^{\rho}}{\rho}\right)^{\alpha}\|\mathfrak{v}-\overline{\mathfrak{v}}\|_{P C_{1-\gamma, \rho}} \\
& \leq\left[\frac{1}{\Gamma(\gamma)}\left(m L^{*}\left(\frac{b^{\rho}}{\rho}\right)^{\gamma-1}+\frac{m L B(\gamma, 1-\beta(1-\alpha))}{\Gamma(1-\beta(1-\alpha))}\left(\frac{b^{\rho}}{\rho}\right)^{\alpha}\right)+\frac{L B(\gamma, \alpha)}{\Gamma(\alpha)}\left(\frac{b^{\rho}}{\rho}\right)^{\alpha}\right]\|\mathfrak{v}-\overline{\mathfrak{v}}\|_{P C_{1-\gamma, \rho}} \\
& =\|\mathfrak{v}-\overline{\mathfrak{v}}\|_{P C_{1-\gamma, \rho}} .
\end{aligned}
$$

This yields that $\mathfrak{P}$ admits a unique fixed point, which is a solution of Eq. (1.1). 


\section{FUS Analysis}

In this section, we exam the FUS for our FDE (1.1). Let $\varphi: I \rightarrow R^{+}$be a continuous function and $\varepsilon>0$. We need the following inequalities:

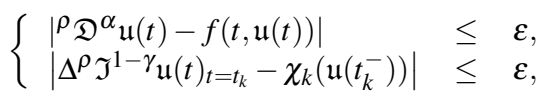

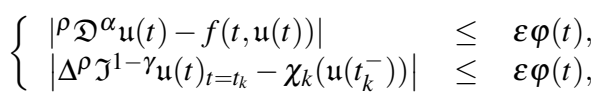

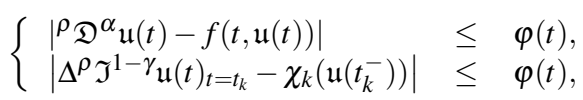

Definition 4.1. The Eq. (1.1) is FUS if there finds a real number $C_{f}>0$ such that for each $\varepsilon>0$ and for each solution $\mathfrak{u} \in P C_{1-\gamma, \rho}(I, R)$ of the inequality (4.1) there exists a solution $\mathfrak{v} \in P C_{1-\gamma, \rho}(I, R)$ of $E q$. (1.1) with

$$
|\mathfrak{u}(t)-\mathfrak{v}(t)| \leq C_{f} \varepsilon, \quad t \in I
$$

Definition 4.2. The Eq. (1.1) is FUS if there occurs a function $\varphi \in P C_{1-\gamma, \rho}(I, R), \varphi_{f}(0)=0$ satisfying that for each solution $\mathfrak{u} \in$ $P C_{1-\gamma, \rho}(I, R)$ of the inequality (4.1) there occurs a solution $\mathfrak{v} \in P C_{1-\gamma, \rho}(I, R)$ of Eq. (1.1) with

$$
|\mathfrak{u}(t)-\mathfrak{v}(t)| \leq \varphi_{f} \varepsilon, \quad t \in I .
$$

Definition 4.3. The Eq. (1.1) is FUHR stable with respect to $\varphi \in P C_{1-\gamma, \rho}(I, R)$ if there occurs a real number $C_{f, \varphi}>0$ such that for each $\varepsilon>0$ and for each solution $\mathfrak{u} \in P C_{1-\gamma, \rho}(I, R)$ of the inequality (4.2) there exists a solution $\mathfrak{v} \in P C_{1-\gamma, \rho}(I, R)$ of Eq. (1.1) with

$$
|\mathfrak{u}(t)-\mathfrak{v}(t)| \leq C_{f, \varphi} \varepsilon \varphi(t), \quad t \in I .
$$

Definition 4.4. The Eq. (1.1) is FUHRS with respect to $\varphi \in P C_{1-\gamma, \rho}(I, R)$ if there finds a real number $C_{f, \varphi}>0$ such that for each solution $\mathfrak{u} \in P C_{1-\gamma, \rho}(I, R)$ of the inequality (4.3) there occurs a solution $\mathfrak{v} \in C_{1-\gamma, \rho}(I, R)$ of Eq. (1.1) with

$$
|\mathfrak{u}(t)-\mathfrak{v}(t)| \leq C_{f, \varphi} \varphi(t), \quad t \in I .
$$

Remark 4.5. A function $\mathfrak{u} \in P C_{1-\gamma, \rho}(I, R)$ is a solution of the inequality (4.1) if and only if there finds a function $g \in P C_{1-\gamma, \rho}(I, R)$ such that

(i) $|g(t)| \leq \varepsilon,\left|g_{k}\right|<\varepsilon, t \in I$.

(ii) $\rho_{\mathfrak{D}^{\alpha, \beta}} \mathfrak{u}(t)=f(t, \mathfrak{u}(t))+g(t), t \in I^{\prime}$.

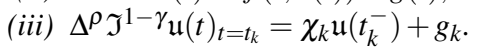

Remark 4.6. If $\mathfrak{u}$ is a solution of the inequality (4.1), then $\mathfrak{u}$ is a solution of the following integral inequality

$$
\begin{aligned}
& \left|\mathfrak{u}(t)-\left(\frac{t^{\rho}-t_{k}^{\rho}}{\rho}\right)^{\gamma-1} \frac{1}{\Gamma(\gamma)}\left[a+\sum_{0<t_{k}<t} \chi_{k} \mathfrak{u}\left(t_{k}\right)+\sum_{0<t_{k}<t} \rho_{I_{a_{+}}^{1-\beta(1-\alpha)}} f\left(t_{k}, \mathfrak{u}\left(t_{k}\right)\right)\right]-{ }^{\rho} I_{a_{+}}^{\alpha} f(t, \mathfrak{u}(t))\right| \\
& \leq \varepsilon\left[\left(\frac{b}{\rho}\right)^{\gamma-1} \frac{1}{\Gamma(\gamma)}\left(m+\frac{m}{\Gamma(2-\beta(1-\alpha))}\left(\frac{b}{\rho}\right)^{1-\beta(1-\alpha)}\right)+\frac{1}{\Gamma(\alpha+1)}\left(\frac{b}{\rho}\right)^{\alpha-1}\right] .
\end{aligned}
$$

Moreover, by Remark 4.5, one can realize that

$$
\begin{aligned}
\rho_{\mathfrak{D}^{\alpha, \beta}} \mathfrak{u}(t) & =f(t, \mathfrak{u}(t))+g(t), t \in I \\
\Delta^{\rho} \mathfrak{I}^{1-\gamma_{\mathfrak{u}}(t)_{t=t_{k}}} & =\chi_{k} \mathfrak{u}\left(t_{k}^{-}\right)+g_{k} .
\end{aligned}
$$

Then

$$
\mathfrak{u}(t)=\left(\frac{t^{\rho}-t_{k}^{\rho}}{\rho}\right)^{\gamma-1} \frac{1}{\Gamma(\gamma)}\left[a+\sum_{0<t_{k}<t} \chi_{k} \mathfrak{u}\left(t_{k}\right)+\sum_{0<t_{k}<t} \rho_{\mathfrak{I}_{a_{+}}^{1-\beta(1-\alpha)}} f\left(t_{k}, \mathfrak{u}\left(t_{k}\right)\right)\right]+{ }^{\rho} \mathfrak{I}_{a_{+}}^{\alpha} f(t, \mathfrak{u}(t)) .
$$

From this it follows that

$$
\begin{aligned}
& \left|\mathfrak{u}(t)-\left(\frac{t^{\rho}-t_{k}^{\rho}}{\rho}\right)^{\gamma-1} \frac{1}{\Gamma(\gamma)}\left[a+\sum_{0<t_{k}<t} \chi_{k} \mathfrak{u}\left(t_{k}\right)+\sum_{0<t_{k}<t} \rho_{\mathfrak{I}_{a_{+}}^{1-\beta(1-\alpha)}} f\left(t_{k}, \mathfrak{u}\left(t_{k}\right)\right)\right]-{ }^{\rho} \mathfrak{I}_{a_{+}}^{\alpha} f(t, \mathfrak{u}(t))\right| \\
& \leq\left(\frac{t^{\rho}-t_{k}^{\rho}}{\rho}\right)^{\gamma-1} \frac{1}{\Gamma(\gamma)}\left[\sum_{0<t_{k}<t}\left|g_{k}\right|+\sum_{0<t_{k}<t} \rho_{\mathfrak{I}_{a_{+}}^{1-\beta(1-\alpha)}}\left|g\left(t_{k}\right)\right|\right]+{ }^{\rho} \mathfrak{I}_{a_{+}}^{\alpha}|g(t)| \\
& \leq \varepsilon\left[\left(\frac{b}{\rho}\right)^{\gamma-1} \frac{1}{\Gamma(\gamma)}\left(m+\frac{m}{\Gamma(2-\beta(1-\alpha))}\left(\frac{b}{\rho}\right)^{1-\beta(1-\alpha)}\right)+\frac{1}{\Gamma(\alpha+1)}\left(\frac{b}{\rho}\right)^{\alpha}\right] .
\end{aligned}
$$

We have similar remarks for the inequality (4.2) and (4.3).

Now, we give the main results, FUHRS results. 
Theorem 4.7. The hypothesis [H1], [H3] and [H5] holds. Then Eq.(1.1) is FUHRS.

Proof. Let $\mathfrak{u}$ be solution of 4.3 and by Theorem 3.2 there $\mathfrak{v}$ is unique solution of the problem

$$
\begin{aligned}
\rho_{\mathfrak{D}^{\alpha, \beta}} \mathfrak{v}(t) & =f(t, \mathfrak{v}(t), \mathfrak{v}(\lambda t)), \quad t \in I, \\
\Delta^{\rho} \mathfrak{I}^{1-\gamma_{\mathfrak{u}}(t)_{t=t_{k}}} & =\chi_{k} \mathfrak{u}\left(t_{k}^{-}\right)+g_{k}, \\
\rho_{\mathfrak{I}^{1-\gamma}} & =\rho_{\mathfrak{v}(0)} \mathfrak{I}^{1-\gamma} \mathfrak{u}(0) .
\end{aligned}
$$

Then, we have

$$
\mathfrak{v}(t)=\left(\frac{t^{\rho}-t_{k}^{\rho}}{\rho}\right)^{\gamma-1} \frac{1}{\Gamma(\gamma)}\left[a+\sum_{0<t_{k}<t} \chi_{k} \mathfrak{v}\left(t_{k}\right)+\sum_{0<t_{k}<t} \rho_{\mathfrak{I}_{a_{+}}^{1-\beta(1-\alpha)}}^{1-\alpha}\left(t_{k}, \mathfrak{v}\left(t_{k}\right)\right)\right]+{ }^{\rho} \mathfrak{I}_{a_{+}}^{\alpha} f(t, \mathfrak{v}(t)) .
$$

By differentiating inequality (4.3), we have

$$
\begin{aligned}
& \left|\mathfrak{u}(t)-\left(\frac{t^{\rho}-t_{k}^{\rho}}{\rho}\right)^{\gamma-1} \frac{1}{\Gamma(\gamma)}\left[a+\sum_{0<t_{k}<t} \chi_{k} \mathfrak{u}\left(t_{k}\right)+\sum_{0<t_{k}<t} \rho_{\mathfrak{I}_{a_{+}}^{1-\beta(1-\alpha)}} f\left(t_{k}, \mathfrak{u}\left(t_{k}\right)\right)\right]-{ }^{\rho} \mathfrak{I}_{a_{+}}^{\alpha} f(t, \mathfrak{u}(t))\right| \\
& \leq\left(\left(\frac{b^{\rho}}{\rho}\right)^{\gamma-1} \frac{m}{\Gamma(\gamma)}\left(1+\lambda_{\varphi}\right)+\lambda_{\varphi}\right) \varphi(t) .
\end{aligned}
$$

Hence, it follows

$$
\begin{aligned}
|\mathfrak{u}(t)-\mathfrak{v}(t)| \leq & \mid \mathfrak{u}(t)-\left(\frac{t^{\rho}-t_{k}^{\rho}}{\rho}\right)^{\gamma-1} \frac{1}{\Gamma(\gamma)}\left[a+\sum_{0<t_{k}<t} \chi_{k} \mathfrak{v}\left(t_{k}\right)+\sum_{0<t_{k}<t} \rho_{\left.\mathfrak{I}_{a_{+}}^{1-\beta(1-\alpha)} f\left(t_{k}, \mathfrak{v}\left(t_{k}\right)\right)\right]-}{ }^{\mathfrak{I}_{a_{+}}^{\alpha}} f(t, \mathfrak{v}(t)) \mid\right. \\
\leq & \mid \mathfrak{u}(t)-\left(\frac{t^{\rho}-t_{k}^{\rho}}{\rho}\right)^{\gamma-1} \frac{1}{\Gamma(\gamma)}\left[a+\sum_{0<t_{k}<t} \chi_{k} \mathfrak{u}\left(t_{k}\right)+\sum_{0<t_{k}<t} \rho_{\left.\mathfrak{I}_{a_{+}}^{1-\beta(1-\alpha)} f\left(t_{k}, \mathfrak{u}\left(t_{k}\right)\right)\right]-{ }^{\rho} \mathfrak{I}_{a_{+}}^{\alpha} f(t, \mathfrak{u}(t)) \mid}\right. \\
& +\left(\frac{t^{\rho}-t_{k}^{\rho}}{\rho}\right)^{\gamma-1} \frac{1}{\Gamma(\gamma)}\left(\left|\chi_{k} \mathfrak{u}\left(t_{k}\right)-\chi_{k} \mathfrak{v}\left(t_{k}\right)\right|+{ }^{\rho} \mathfrak{I}_{a_{+}}^{1-\beta(1-\alpha)}\left|f\left(t_{k}, \mathfrak{u}\left(t_{k}\right)\right)-f\left(t_{k}, \mathfrak{v}\left(t_{k}\right)\right)\right|\right) \\
& +{ }^{\rho} \mathfrak{I}_{a_{+}}^{\alpha}|f(t, \mathfrak{u}(t))-f(t, \mathfrak{v}(t))| \\
\leq & \left(\left(\frac{b^{\rho}}{\rho}\right)^{\gamma-1} \frac{m}{\Gamma(\gamma)}\left(1+\lambda_{\varphi}\right)+\lambda_{\varphi}\right) \varphi(t)+\left(\frac{b^{\rho}}{\rho}\right)^{\gamma-1} \frac{L_{k}^{*}}{\Gamma(\gamma)}\left|\mathfrak{u}\left(t_{k}\right)-\mathfrak{v}\left(t_{k}\right)\right| \\
& +\left[\frac{m}{\Gamma(\gamma)}\left(\frac{b^{\rho}}{\rho}\right)^{\alpha} \frac{m L}{\Gamma(2-\beta(1-\alpha))}+\frac{L}{\Gamma(\alpha)}\left(\frac{b^{\rho}}{\rho}\right)^{\alpha}\right]|\mathfrak{u}(t)-\mathfrak{v}(t)| .
\end{aligned}
$$

By the properties, there occurs a constant $M^{*}>0$ independent of $\lambda_{\varphi} \varphi(t)$ such that

$$
|\mathfrak{u}(t)-\mathfrak{v}(t)| \leq M^{*} \lambda_{\varphi} \varphi(t):=C_{f, \varphi} \varphi(t) .
$$

Thus, Eq.(1.1) is FUHRS.

\section{References}

[1] M. I. Abbas, Ulam stability of fractional impulsive differential equations with Riemann-Liouville integral boundary conditions Journal of Contemporary Mathematical Analysis, 2015, (50), 209-219.

[2] M. Benchohra, B. Slimani, Existence and uniqueness of solutions to impulsive fractional differential equations, Electronic Journal of Differential Equations, 10, (2009), 1-11.

[3] K. M. Furati, M. D. Kassim, N. E. Tatar, Existence and uniqueness for a problem involving hilfer fractional derivative, Computer and Mathematics with Application, 64, (2012), 1616-1626.

[4] R. Hilfer, Applications of Fractional Calculus in Physics, World scientific, Singapore, 1999.

[5] R.W. Ibrahim, H.A. Jalab , Existence of Ulam stability for iterative fractional differential equations based on fractional entropy, Entropy 17 (5) (2015) 3172 .

[6] R.W. Ibrahim, Ulam-Hyers stability for Cauchy fractional differential equation in the unit disk, Abstract Appl. Anal. (2012) 1.

[7] R.W. Ibrahim, Generalized Ulam-Hyers stability for fractional differential equations, Int. J. Math. 23 (05) (2012) 1.

[8] R.W. Ibrahim, Ulam stability for fractional differential equation in complex domain, Abstract Appl. Anal. (2012) 1.

[9] R. Kamocki, C. Obczynski, On fractional Cauchy-type problems containing Hilfer's derivative, Electronic Journal of Qualitative Theory of Differential Equations, 2016, 50, 1-12.

[10] R. Kamocki, A new representation formula for the Hilfer fractional derivative and its application, Journal of Computational and Applied Mathematics, $308,(2016), 39-45$.

[11] U.N. Katugampola, Existence and uniqueness results for a class of generalized fractional differential equations, Bulletin of Mathematical Analysis and Applications, arXiv:1411.5229, v1 (2014). https://arxiv.org/abs/1411.5229.

[12] V. Lakshmikantham, D. D. Bainov, P. S. Simeonov, Theory of Impulsive Differential Equations, World scientific, Singapore(1989).

[13] X. Liu, Y. Li, Some Antiperiodic Boundary Value Problem for Nonlinear Fractional Impulsive Differential Equations, Abstract and Applied Analysis, (2014).

[14] Z. Luo, J. Shen, Global existence results for impulsive functional differential equation, Journal of Kathematical Analysis and Application, 323, (2006),

[15] D4. S. Oliveira, E. Capelas de oliveira, Hilfer-Katugampola fractional derivative, arxiv:1705.07733v1, 2017.

[16] A. Ouahab, Local and global existence and uniqueness results for impulsive differential equations with multiple delay, Journal of Mathematical Analysis and Application, 323, (2006), 456-472.

[17] J. Wang, L. Lv, Y. Zhou, Ulam stability and data dependence for fractional differential equations with Caputo derivative, Electronic Journal of Qualitative Theory of Differential Equations, 63, (2011), 1-10. 